\title{
Учение Е.К. Войшвилло о понятии: значение и перспективы
}

\author{
В. И. МАРКИН
}

\begin{abstract}
The paper contains the analysis of the Theory of Concept in its modern version worked out by E.K. Voishvillo. Its advances in the study of concept as a form of thought are observed. At the same time some points for further research are marked out.

Keywords: concept, extension, intension, genus, predicate, theory, entailment, logical form, formalized language
\end{abstract}

Одним из наиболее важных достижений Евгения Казимировича Войшвилло в логике была разработка им современного варианта учения о понятии. Именно за его создание он получил высшую научную награду Московского университета - Ломоносовскую премию первой степени.

Основы учения о понятии были заложены еще Аристотелем. В развитом виде оно сформировалось в рамках традиционной логики и вошло в качестве важнейшего, фундаментального раздела во все университетские курсы логики и учебники по формальной логике. Обучение логике стало начинаться именно с анализа понятий. Во многом эта традиция в нашей стране сохранилась и поныне, особенно при чтении коротких курсов логики для студентов нефилософских специальностей.

Однако после революции в логической науке конца XIXначала XX в., после создания математической логики проблематика, связанная с изучением понятий, на долгое время отошла на второй план (лишь в последнее время наблюдается рост интереса к этой проблематике в связи с вопросами представления знаний, разрабатываемыми в рамках программы искусственного интеллекта). Это объяснялось, во-первых, доминированием 
номиналистической и антиметафизической установок у основателей современной логики. А во-вторых, само учение о понятии в его традиционном варианте было недостаточно проработанным, не отвечало новым логическим критериям строгости и содержало массу пробелов и внутренних несоответствий.

Непосредственный мотив, побудивший Е.К. Войшвилло провести ревизию учения о понятии, был связан, по-видимому, с необходимостью органичного сочетания проблематики традиционной и символической логики при чтении фундаментального общего курса логики для студентов-философов. Данный вопрос имел принципиальное значение и оставлял несколько вариантов для выбора. Можно ограничиться чтением традиционной логики, пополнив ее изложение некоторыми элементами символической. Ясно, что адекватных представлений о современном состоянии логики как науки подобный курс дать не способен. Альтернативный подход состоит в чтении именно символической логики, где о предшествующем ей этапе говориться вскользь, в основном в историческом аспекте. В этом случае, по существу, игнорируются достижения логики за всю ее многовековую историю за исключением последних 100-150 лет. Иногда два описанных выше подхода механически сочетают в рамках одного курса: сначала знакомят студентов с проблематикой традиционной логики, а затем преподают основы символической логики. Однако при этом не выявляется единство логики как науки, более того, иногда даже заявляется тезис о том, что традиционная и символическая логика - это вообще две разные науки.

Идея курса логики, разработанного и читавшегося Евгением Казимировичем на философском факультете МГУ, была принципиально иная. Логика представлялась студентам как единая наука, без разделения на традиционную и современную. Подчеркивалась неизменность, в главных чертах, предмета логики, но в то же время фиксировались те изменения в логических методах, которые происходили на протяжении всей ее истории. Особый упор делался на демонстрации значения и преимуществ современных методов, которые сделали логику подлинно теоретической наукой. Вся «традиционная» проблематика включалась в 
состав курса, но рассматривалась там сквозь призму результатов и методов символической логики.

Такой подход требовал внесения радикальных изменений в преподавание, в том числе и раздела «Понятие». Это и побудило, на мой взгляд, Евгения Казимировича создать современную версию логического учения о понятии, лишенную пробелов и внутренней несогласованности старого варианта данного учения. Он успешно применил к анализу этой «традиционной» проблемы инструментарий, разработанный в символической логике, прежде всего метод формализованных языков, логических исчислений, а также логико-семантические методы анализа естественного языка.

Первое подробное изложение современной версии учения о понятии содержится в фундаментальной монографии Е.К. Войшвилло «Понятие» [3], вышедшей в 1967 году. Эта книга по праву стала классическим трудом в отечественной логикофилософской школе и, по-видимому, самым известным научным изданием по логике в нашей стране в ХХ столетии. Известность и популярность этой монографии во многом объясняли тем, что она - в отсутствие качественных учебников по современной философской логике - широко использовалась многими поколениями студентов-философов в качестве базового учебного пособия по курсу логики. Сжатое изложение основных положений данного учения было опубликовано в том же 1967 году в специальном подразделе энциклопедической статьи «Понятие» в 4-м томе «Философской энциклопедии» [4].

Более чем через два десятилетия, в 1989 году Е.К. Войшвилло возвращается к этой проблематике и публикует монографию «Понятие как форма мышления: логико-гносеологический анализ» [5], в которой детализирует и развивает свое учение. Достаточно полное, но вместе с тем и лаконичное изложение этого учения можно встретить в фундаментальном учебнике Е.К. Войшвилло и М.Г. Дегтярева [6].

Следует с сожалением отметить, что оригинальная концепция понятия Е.К. Войшвилло осталась, в отличие от других его научных результатов, практически незамеченной мировым логическим сообществом. Авторские работы по данной теме на англий- 
ском языке отсутствуют. Единственная публикация на европейском языке - перевод монографии «Понятие» на венгерский, осуществленный ученицей Е.К. Войшвилло Каталин Хаваш.

Непростой оказалась судьба этой концепции и в отечественной логике. Здесь также редко встретишь публикации, в которых учение о понятии Е.К. Войшвилло развивается, уточняется или подвергается критическому анализу. Причина такого положения дел видится в следующем. Глубина проработанности концепции E.K. Войшвилло, основательность и продуманность основных ее положений, а также непререкаемый научный и личностный авторитет автора задали столь высокую планку, что логики последующих поколений «не рисковали» творчески работать в этом проблемном поле или не видели научных перспектив такой работы. В результате данная концепция лишь излагается (иногда с небольшими модификациями) в отдельных учебниках по логике, написанных коллегами и учениками Евгения Казимировича (см., например, [1], [7]). Более того, последние годы в научных и учебных публикациях, так или иначе затрагивающих проблематику понятий, концепция Е.К. Войшвилло практически игнорируется, и достаточно часто в них воспроизводится традиционная трактовка понятия со всеми ее недочетами, а порой и откровенными нелепостями.

100-летний юбилей Евгения Казимировича дает нам хороший повод снова вернуться к разработанной им современной концепции понятия, но не как к музейному экспонату, а как к живому, развивающемуся учению. В данной статье я попытаюсь, во-первых, выделить основные положения этой концепции, знаменовавшие прорыв в исследовании понятия как формы мысли, а во-вторых, указать на некоторые проблемные места в этом учении, требующие, на мой взгляд, определенных корректировок, уточнений и дальнейшего теоретического осмысления.

Главной находкой Е.К. Войшвилло оказался сам исходный пункт его учения о понятии - знаковое представление понятия (и его логической формы) в виде конструкции одного из двух типов: $\alpha A(\alpha)$, где $\alpha$ представляет собой переменную, а $A(\alpha)-$ одноместный предикат, содержащий $\alpha$ в качестве свободной переменной, либо $\alpha_{1} \alpha_{2} \ldots \alpha_{n} A\left(\alpha_{1}, \alpha_{2}, \ldots, \alpha_{n}\right)$, где $\alpha_{1}, \alpha_{2}, \ldots, \alpha_{n}$ - пе- 
ременные, а $A\left(\alpha_{1}, \alpha_{2}, \ldots, \alpha_{n}\right)-n$-местный предикат со свободными переменными $\alpha_{1}, \alpha_{2}, \ldots, \alpha_{n}$. Понятия первого типа собирают в класс (обобщают) отдельно взятые объекты (например, «человек, родившийся в 1900 году»), а понятия второго типа - кортежи, то есть пары, тройки и т.д. объектов (например, «люди, родившиеся в одном и том же году»).

Эти конструкции соответствуют дефиниенсу родо-видовых определений, который в естественном языке выражается посредством описательного термина вида «объект (кортеж объектов) из универсума $\mathbf{U}$, обладающий признаком $A »$. А ведь именно дефиниенс как раз и выражает подлинное понятие - смысл, связываемый с неописательным термином (дефиниендумом).

Конструкция $\alpha A(\alpha)$ (как и $\left.\alpha_{1} \alpha_{2} \ldots \alpha_{n} A\left(\alpha_{1}, \alpha_{2}, \ldots, \alpha_{n}\right)\right)$ может быть определена в рамках стандартного языка логики предикатов в качестве особого, дополнительного - наряду с термами и формулами - типа правильно построенных выражений. Причем выделение выражения $\alpha A(\alpha)$ как играющего специфическую семантическую и синтаксическую роль имеет под собой достаточно серьезные основания, никак не связанные с учением о понятии. Например, Е.Д. Смирнова, ставя целью установить категорию кванторов $\forall$ и $\exists$ (именно самих кванторов, а не кванторных комплексов $\forall \alpha$ и $\exists \alpha$ ), попутно приходит к тому, что выражение $\alpha A(\alpha)$ имеет свою семантическую категорию: если $A$ - выражение категории $s$, а $\nu$ - переменная категории $\beta$, и при этом $\nu$ входит свободно в $A$, то $\nu A$ является выражением категории $s / \beta[9$, с. 71]. В частности, если $\alpha-$ предметная (индивидная) переменная, то языковое выражение $\alpha A(\alpha)$ имеет категорию $s / n$.

Предложенные Е.К. Войшвилло знаковые понятийные конструкции дают возможность точной записи как самого понятия (в прикладном языке логики предикатов), так и его логической формы. В последнем случае конкретные имена, предикаторы и предметные функторы заменяются параметрами (константами) соответствующего типа из алфавита языка «чистой» логики предикатов. Например, понятие о парах параллельных прямых в прикладном языке можно выразить так: $x y(x-$ прямая $\wedge y-$ прямая $\wedge \exists z(z-$ плоскость $\wedge x$ лежит в $z \wedge y$ лежит в 
$z) \wedge(\neg \exists z(z-$ точка $\wedge z$ принадлежит $x \wedge z$ принадлежит $y) \vee$ $x$ совпадает с $y)$ ). Логическая форма данного понятия может быть выражена в «чистом» первопорядковом языке следующим образом: $x y(P(x) \wedge P(y) \wedge \exists z(Q(z) \wedge R(x, z) \wedge R(y, z)) \wedge(\neg \exists z(S(z) \wedge$ $\left.\left.\left.R_{1}(z, x) \wedge R_{1}(z, y)\right) \vee R_{2}(x, y)\right)\right)$.

Понятийные конструкции Е.К. Войшвилло позволяют задать точные современные аналоги таких традиционных характеристик понятия, как его род, объем и содержание.

Родом (универсумом) понятия $\alpha A(\alpha)$ является множество, представляющее собой область пробега переменной $\alpha$, а родом понятия $\alpha_{1} \alpha_{2} \ldots \alpha_{n} A\left(\alpha_{1}, \alpha_{2}, \ldots, \alpha_{n}\right)$ - декартово произведение областей пробега переменных $\alpha_{1}, \alpha_{2}, \ldots, \alpha_{n}$.

Конструкция $\alpha A(\alpha)$ не является термом-дескрипцией, так как она не содержит оператора дескрипции. Это, по словам Е.К. Войшвилло, специфицированная переменная, сужающая область пробега обычной, неспецифицированной переменной $\alpha$. Сужение области пробега происходит со всего универсума (предметной области) до его подмножества $\{\alpha: A(\alpha)\}$. Это подмножество (обозначаемое Е.К. Войшвилло также посредством $\mathbf{W} \alpha A(\alpha))$ представляет собой не что иное, как объем понятия $\alpha A(\alpha)$, то есть множество предметов, выделяемых из универсума и обобщаемых в данном понятии. Объемом понятия $\alpha_{1} \alpha_{2} \ldots \alpha_{n} A\left(\alpha_{1}, \alpha_{2}, \ldots, \alpha_{n}\right)$ будет множество $n$-ок, компоненты которых берутся из областей значений соответствующих переменных $\left\{<\alpha_{1}, \alpha_{2}, \ldots, \alpha_{n}>\right.$ : $\left.A\left(\alpha_{1}, \alpha_{2}, \ldots, \alpha_{n}\right)\right\}$, обозначаемое также в виде $\mathbf{W}<\alpha_{1}, \alpha_{2}, \ldots, \alpha_{n}>A\left(\alpha_{1}, \alpha_{2}, \ldots, \alpha_{n}\right)$.

Что же касается другой основной характеристики понятия - его содержания, то его знаковым представлением Е.К. Войшвилло предложил считать сам предикат $A(\alpha)$ (либо $\left.A\left(\alpha_{1}, \alpha_{2}, \ldots, \alpha_{n}\right)\right)$. Это хорошо согласуется с распространенной трактовкой содержания понятия как признака, на основе которого происходит выделение из универсума объектов, этим признаком обладающих. Ведь именно предикаты - высказывательные формы со свободными переменными - являются способом выражения признаков в языке.

Хочу обратить внимание на то, что предлагаемая Е.К. Войшвилло экспликация содержания понятия как предиката не 
вполне сочетается с даваемым им же определением термина «содержание понятия». Под последним, он, как и обычно в традиционной логике, понимает «совокупность признаков, по которым обобщаются предметы в понятия» (см., например, [5, с. 92]). Но предикат не может быть знаковым выражением совокупности, он попросту не репрезентирует множество! K тому же трактовка содержания понятия как совокупности признаков приводит к явным затруднениям (на некоторые из них сам Войшвилло обращает внимание, исследуя закон обратного отношения). Дело ведь не только в том, какие признаки имеются в содержании понятия, но и как они связаны там между собой.

Поэтому более естественной выглядит трактовка содержания понятия не как совокупности признаков, а как просто признака, по которому понятие выделяет и обобщает предметы в класс. При этом, конечно, требуется оговорка, что сами признаки могут быть не только простыми, но и сложными. Кстати, указанное обстоятельство сам Войшвилло неоднократно подчеркивает при изложении своего учения.

Одним из центральных пунктов учения о понятии Е.К. Войшвилло является тезис о необходимости различения логических и фактических содержаний, а также логических и фактических объемов понятий.

Дистинкция логического и фактического содержания успешно осуществляется с использованием понятийной языковой конструкции $\alpha A(\alpha)^{1}$. «Фактическое содержание - это информация, которую мы имеем в понятии с учетом значений имеющихся в его формулировке дескриптивных терминов (знаков предметов, свойств, отношений)» [5, с. 139]. Иными словами, фактическим содержанием понятия $\alpha A(\alpha)$ является та информация, которую имеет предикат $A(\alpha)$, сформулированный в прикладном языке, с учетом значений входящих в его состав нелогических терминов. Учет значения таких терминов, согласно Е.К. Войшвилло, означает принятие во внимание некоторой совокупности знаний Г относительно предметов, свойств и отношений, знаками кото-

\footnotetext{
${ }^{1} \mathrm{~B}$ этой части работы я не рассматриваю для простоты конструкцию $\alpha_{1} \alpha_{2} \ldots \alpha_{n} A\left(\alpha_{1}, \alpha_{2}, \ldots, \alpha_{n}\right)$, однако все рассуждения справедливы и для нее.
} 
рых эти термины являются. В роли $\boldsymbol{\Gamma}$ может выступать, например, научная теория, в которой понятие $\alpha A(\alpha)$ используется.

Логическое содержание понятия $\alpha A(\alpha)$ рассматривается как результат отвлечения от того, какие именно значения имеют содержащиеся в выражении $A(\alpha)$ нелогические термины. Поэтому логическим содержанием данного понятия будет та информация, которую несет логическая форма выражения $A(\alpha)$.

Например, фактическим содержанием понятия «натуральное число, кратное 2 и 3» является признак «быть кратным 2 и 3 » при учете тех значений, которые имеют в арифметике имена «2» и «3», а также знак отношения «кратно». Логическим же содержанием будет та информация, которую выражает формула $R(x, a) \wedge R(x, b)$, то есть признак «находиться в одном и том же отношении к двум возможно различным предметам».

Фактический обгем понятия $\alpha A(\alpha)$ Е.К. Войшвилло определяет как множество фактически существующих объектов, удовлетворяющих условию $A$ с учетом значений дескриптивных терминов в составе $A(\alpha)$. Здесь, конечно, речь идет не обязательно о реально существующих предметах нашего, «действительного» мира, а о существовании объектов в исходной предметной области, в универсуме (роде) понятий. Сам этот универсум может содержать как реальные предметы (людей, планеты, здания), так и идеальные объекты (числа, материальные точки, характеристики предметов, отделенные от самих этих предметов и т.п.), и даже фикции типа мифологических существ или литературных персонажей. Важно лишь, что данный универсум составляют вполне определенные объекты мысли, а фактический объем выделяется из универсума фактическим содержанием понятия, то есть конкретным признаком (например, «быть кратным 2 и $3 \gg)$, которым обладают эти выделяемые объекты.

Несколько сложнее обстоит дело с трактовкой логического объема понятия. Сам Е.К. Войшвилло понимает под ним множество возможных объектов, выполняющих предикат $A(\alpha)$ без учета значений имеющихся в нем дескриптивных терминов (то есть при рассмотрении выражения $A(\alpha)$ исключительно с точки зрения его логической формы) [5, с. 140]. Такая трактовка кажется естественной. Однако возникают вопросы: какова приро- 
да этих «возможных объектов», из чего в данном случае состоит исходный универсум (род) понятия? Также из людей, планет, зданий, чисел, материальных точек, характеристик предметов, мифологических существ, литературных персонажей и других подобных им объектов?

Следует заметить, что в работах Е.К. Войшвилло приводятся примеры установления логических объемов только для таких понятий, содержания которых не имеют в своем составе кванторов. По существу, логический объем тогда можно выделить с использованием стандартных диаграмм Венна. В них для решения этой задачи конструируется особый универсум - множество обезличенных абстрактных «точек». Простые признаки задаются на данном универсуме независимо друг от друга, т.е. для любых простых признаков $P_{1}(\alpha), P_{2}(\alpha), \ldots, P_{n}(\alpha)$ в составе $A(\alpha)$ пересечение множеств $\mathbf{W} \alpha P_{1}(\alpha)^{*}, \mathbf{W} \alpha P_{2}(\alpha)^{*}, \ldots, \mathbf{W} \alpha P_{n}(\alpha)^{*}$ (где $\mathbf{W} \alpha P_{i}(\alpha)^{*}$ есть либо само $\mathbf{W} \alpha P_{i}(\alpha)$, либо дополнение к нему), полагается непустым. Каждое множество $\mathbf{W} \alpha P_{i}(\alpha)^{*}$ также трактуется как непустое. При этом $P_{1}, P_{2}, \ldots, P_{n}$ не являются знаками конкретных характеристик объектов, а играют роль абстрактных параметров этих характеристик. Под логическим объемом понятия подразумевается подмножество сконструированного указанным образом универсума, которое выделяется логическим содержанием понятия. Что же касается вопроса о логическом объеме понятия $\alpha A(\alpha)$ для тех случаев, когда $A(\alpha)$ содержит кванторы, то он еще требует своего решения с привлечением более изощренных теоретико-модельных конструкций.

Дистинкция фактических и логических объемов и содержаний позволяет существенным образом уточнить формулировку знаменитого закона традиционной логики - закона обратного отношения между содержаниями и обгемами понятий. Данный закон - в обычной, «традиционной» своей формулировке неоднократно подвергался справедливой критике, которая, по существу, лишала его статуса закона. Однако осуществленное E.K. Войшвилло различение логических и фактических характеристик понятия позволило снять все эти возражения. Это достигается посредством существенного уточнения: утверждения «объем первого понятия есть часть объема второго» и «содержа- 
ние второго понятия есть часть содержания первого» должны оба относиться либо к фактическим объемам и содержаниям, либо к логическим. Известные «контрпримеры» к данному закону как раз и основываются на смешении логического и фактического аспектов: отношение между объемами рассматривается с фактической точки зрения, а отношение между содержаниями с логической, либо наоборот.

Отношение «быть частью объема» соответствует обычному теоретико-множественному включению, в то время как сравнение содержаний понятий на предмет того, является ли одно частью второго, осуществляется с использованием отношения логического следования между соответствующими предикатами.

Для фактических характеристик понятий $\alpha A(\alpha)$ и $\alpha B(\alpha)$ Е.К. Войшвилло формулирует закон обратного отношения так:

$\mathbf{W} \alpha A(\alpha) \subseteq \mathbf{W} \alpha B(\alpha)$, если и только если $\boldsymbol{\Gamma}, A(\alpha) \models B(\alpha)$,

где левая часть эквивалентности означает, что фактический объем первого понятия ( $\mathbf{W} \alpha A(\alpha))$ есть часть фактического объема второго $(\mathbf{W} \alpha B(\alpha))$, а правая ее часть утверждает, что фактическое содержание второго понятия $(B(\alpha))$ есть часть фактического содержания первого $(A(\alpha))$. При этом $A(\alpha)$ и $B(\alpha)$ представляют собой выражения прикладного языка логики предикатов, а $\boldsymbol{\Gamma}$, как уже говорилось выше, есть некоторая совокупность истинных высказываний о предметах, свойствах и отношениях в соответствующем предметном поле.

Для логических объемов и содержаний закон обратного отношения действует в несколько иной формулировке:

$\mathbf{W} \alpha A(\alpha) \subseteq \mathbf{W} \alpha B(\alpha)$, если и только если $A(\alpha)=B(\alpha)$.

В данном случае множество $\boldsymbol{\Gamma}$ пусто, $A(\alpha)$ и $B(\alpha)$ - логические формы языковых выражений, соответствующих содержаниям исследуемых понятий, а $\mathbf{W} \alpha A(\alpha)$ и $\mathbf{W} \alpha B(\alpha)$ - их логические объемы, то есть подмножества универсума абстрактно возможных объектов, выделяемые на основе той информации, которую содержат указанные логические формы.

Отношение логического следования (и его синтаксический аналог - отношение выводимости) Е.К. Войшвилло использует 
для введения еще одного важного конструкта - полного содержания понятия. Признак $A(\alpha)$ в понятии $\alpha A(\alpha)$ может трактоваться как основное содержание данного понятия, а совокупность всех признаков, которые можно «извлечь», «дедуцировать», «вывести» из него (возможно, с учетом множества знаний $\boldsymbol{\Gamma})$ - как полное его содержание. Для полного содержания понятия, как и для основного, Е.К. Войшвилло вводит различение фактического и логического содержания.

Этот конструкт требует, по-видимому, более точной экспликации. Речь ведь идет не о всех логических следствиях из $A(\alpha)$ (возможно, вместе с $\boldsymbol{\Gamma}$ ), а о только о тех, которые выражают признаки выделяемых в понятии предметов. Тогда полное фактическое содержание понятия $\alpha A(\alpha)$ следует определять как множество всех таких предикатов $B(\alpha)$, содержащих свободно в точности одну переменную $\alpha$, для которых верно: $\boldsymbol{\Gamma}, A(\alpha) \models$ $B(\alpha)$. А полным логическим содержанием понятия $\alpha A(\alpha)$ следует называть множество всех формул языка «чистой» логики предикатов, содержащих единственную свободную переменную $\alpha$ и следующих из логической формы выражения $\alpha A(\alpha)$.

Введение в сферу рассмотрения учения о понятии полного содержания позволяет вписать в его рамки, по крайней мере частично, проблематику современной теории определимости. Явную определимость в теории $\boldsymbol{\Gamma}$ произвольного $n$-местного предикаторного символа $P$ можно было бы трактовать так: в языке данной теории существует предикат $A$, не содержащий символа $P$ и содержащий в точности $n$ различных свободных переменных $\alpha_{1}, \alpha_{2}, \ldots, \alpha_{n}$, такой, что полные фактические содержания понятий $\alpha_{1} \alpha_{2} \ldots \alpha_{n} P\left(\alpha_{1}, \alpha_{2}, \ldots, \alpha_{n}\right)$ и $\alpha_{1} \alpha_{2} \ldots \alpha_{n} A$ совпадают.

Ряд существенных уточнений Е.К. Войшвилло внес в один из важнейших разделов учения о понятии, касающийся отношений между понятиями.

Прежде всего хочу обратить внимание на предложенную им четкую и адекватную трактовку сравнимости понятий: понятия сравнимы, если и только если у них один и тот же род (универсум $)^{2}$. Это означает, что логические отношения устанавли-

\footnotetext{
${ }^{2} \mathrm{~K}$ сожалению, до сих пор из учебника в учебник кочуют такие маловразумительные описания сравнимых понятий, как имеющих общие признаки
} 
ваются только между понятиями с одинаковым родом. Если же у исследователя есть необходимость в установлении отношения между понятиями с различными родами, то он должен осуществить процедуру приведения их к общему роду: произвести выбор общего родового термина, а старые родовые термины конъюнктивно присоединить в качестве дополнительных предикатов к видовым отличиям понятий. Важно только понимать, что в результате мы получим другие, модифицированные понятия, хоть и с теми же самыми объемами.

Такая трактовка сравнимых понятий позволяет уточнить характер операций обобщения и ограничения (они осуществляются в рамках одного рода), а также действие закона обратного отношения, под который подпадают именно понятия с одинаковым родом.

Вообще, указание рода при выделении множества предметов на основе обладания некоторым признаком, существенно не только в учении о понятии. У Е.К. Войшвилло есть интересное замечание о важности фиксации рода и в теории множеств, где образование класса по признаку может в некоторых ситуациях, как известно, иметь парадоксальные следствия. Непарадоксальная формулировка аксиомы свертывания $\exists y \forall x(x \in y \equiv \exists z(x \in$ $z \wedge A(x))$, в отличие от парадоксальной $-\exists y \forall x(x \in y \equiv A(x))$, требует, чтобы класс $y$ по признаку $A$ обязательно выделялся в рамках другого класса $z$. А аксиому выделения $\forall z \exists y \forall x(x \in y \equiv$ $(x \in z \wedge A(x))$, согласно Войшвилло, «можно истолковать как указание на то, что всякое понятие $x A(x)$ имеет объем. Класс предметов $z$ в указанной формулировке аксиомы - это область $\mathbf{D}$ значений $x$ (род данного понятия), а $y$ есть не что иное, как именно объем данного понятия» [5, с. 95].

Вопрос о классификации отношений по объему между сравнимыми понятиями излагается Е.К. Войшвилло вполне традиционно, в духе стандартных учебников по логике (см., например, [5, с. 184]). Этот традиционный подход имеет ряд очевидных недостатков.

в содержаниях или имеющие элементы объемов, которые обладают некоторыми одинаковыми признаками. 
Во-первых, по непонятной причине для разновидностей объемной совместимости универсум понятий не фиксируется, а для разновидностей несовместимости фиксируется. Следствием этого является неразличение двух типов перекрещивания: когда объединение объемов перекрещивающихся понятий совпадает с универсумом, и когда не совпадает. В то же время подобный критерий «исчерпываемости объемами универсума» применяется для различения отношений противоречия и соподчинения. В этом аспекте приводимая классификация непоследовательна.

Во-вторых, трактовка отношений равнообъемности и логического подчинения как разновидностей отношения совместимости может быть правомерной только при исключении из сферы рассмотрения пустых понятий. Ведь любые два пустые понятия имеют одинаковый объем, и любое пустое понятие строго включается по объему в любое непустое. Но ни о какой совместимости пустого понятия с любым другим речи ведь идти не может. И следует ли в вопросе о логических отношениях изначально игнорировать пустые понятия?

Третье замечание связано с тем, что экстенсиональными средствами невозможно отличить отношение соподчинения от отношения противоположности. И изображение объемов противоположных понятий в виде кругов на «полюсах» в универсумеэллипсе является не более, чем метафорой, и не отменяет того факта, что данному отношению нельзя сопоставить адекватное теоретико-множественное описание.

Иной - лишенный указанных недостатков - подход к типологизации объемных отношений между понятиями развит В.А. Бочаровым и В.И. Маркиным в [1]. Основная идея состоит в первоначальном выделении трех фундаментальных отношений между любыми двумя понятиями $\alpha A(\alpha)$ и $\alpha B(\alpha)$ по объему: отношения совместимости (в объемах понятий имеется по крайней мере один общий элемент $-\mathbf{W} \alpha A(\alpha) \cap \mathbf{W} \alpha B(\alpha) \neq \emptyset)$, отношения исчерпываемости (объединение объемов совпадает с родом - $\mathbf{W} \alpha A(\alpha) \cup \mathbf{W} \alpha B(\alpha)=\mathbf{U})$ и отношения включения (каждый элемент объема первого понятия является элементом объема второго $-\mathbf{W} \alpha A(\alpha) \subseteq \mathbf{W} \alpha B(\alpha))$. 
Остальные объемные отношения можно рассматривать как комбинации наличия или отсутствия фундаментальных отношений. Более точно: если мы адекватно и в полной мере хотим установить объемное отношение между $\alpha A(\alpha)$ и $\alpha B(\alpha)$, нам следует ответить на четыре вопроса: (1) совместимы ли эти понятия, (2) находятся ли они в отношении исчерпываемости, (3) включается ли $\alpha A(\alpha)$ в $\alpha B(\alpha),(4)$ включается ли $\alpha B(\alpha)$ в $\alpha A(\alpha)$. В результате мы получим возможность зафиксировать соответствующее отношение на характеристической диаграмме. Всего существует 16 таких характеристических диаграмм. Одну из них обычно не рассматривают, так как она предполагает пустоту универсума (рода) понятий. 7 диаграмм характеризуют различные виды отношений между непустыми и неуниверсальными понятиями. Именно эти 7 диаграмм используются в качестве модельных схем в традиционной силлогистике.

Вопрос о видах логических отношений между понятиями по содержанию разработан в логике в гораздо меньшей степени. В работах E.K. Войшвилло можно обнаружить оригинальный подход к решению указанной проблемы. Поскольку содержания и объемы понятий находятся в тесной взаимозависимости, то отношения между понятиями, изначально определяемые как объемные, могут, в принципе, быть охарактеризованы интенсионально, исходя из их содержаний. Иными словами, известные виды отношений между $\alpha A(\alpha)$ и $\alpha B(\alpha)$ могут быть заданы как в теоретико-множественных терминах с использованием классов $\mathbf{W} \alpha A(\alpha)$ и $\mathbf{W} \alpha B(\alpha)$, так и через отношения между предикатами (высказывательными формами) $A(\alpha)$ и $B(\alpha)$, которые фиксируют в языке содержания этих понятий.

Так, поскольку наличие отношения объемного включения первого понятия во второе $(\mathbf{W} \alpha A(\alpha) \subseteq \mathbf{W} \alpha B(\alpha))$ равносильно, в соответствии с известным законом, наличию отношения логического следования $(A(\alpha) \models B(\alpha))$, то можно считать, что понятия $\alpha A(\alpha)$ и $\alpha B(\alpha)$ находятся в указанном отношении, но оно может быть охарактеризовано и в экстенсиональном, и в интенсиональном аспекте.

Развивая данный подход, можно выделить адекватные «содержательные» аналоги других фундаментальных отношений 
между понятиями. Понятия $\alpha A(\alpha)$ и $\alpha B(\alpha)$ являются совместимыми тогда и только тогда, когда высказывательные формы $A(\alpha)$ и $B(\alpha)$ совместимы по истинности. Понятия $\alpha A(\alpha)$ и $\alpha B(\alpha)$ находятся в отношении исчерпываемости, если и только если $A(\alpha)$ и $B(\alpha)$ несовместимы по ложности. Подобные трактовки могут быть сопоставлены всем объемным диаграммам, каждая из которых дает максимально полную и уникальную информацию об отношениях между двумя понятиями.

Выше речь шла об отношениях между понятиями по логическим содержаниям. Для сравнения их фактических содержаний (учитывающих совокупность знаний $\boldsymbol{\Gamma}$ ) требуется ввести ограничение на класс моделей: вопрос об отношениях по истинности и ложности между предикатами $A(\alpha)$ и $B(\alpha)$ следует ставить применительно к моделям, где истинны все предложения из $\boldsymbol{\Gamma}$.

Разработанная Е.К. Войшвилло трактовка понятий позволила ему уточнить критерии для выделения различных их типов.

Различение универсальных и неуниверсалъных понятий проводится на основании того, совпадает ли объем понятия с его родом (универсумом). Например, понятие «смертный человек» оценивается как универсальное (поскольку все люди смертны), а понятие «грамотный человек» как неуниверсальное (не все люди грамотны). Понимание универсальных понятий как таких, в которых объем и род совпадают, гораздо адекватнее распространенных туманных трактовок их как категориальных, предельно общих, охватывающих все и вся, тем более что так называемые понятия-категории на поверку вообе оказываются зачастую единичными (например, понятие материи).

Очень важным является выделение среди универсальных понятий логически универсальных, а среди пустых - логически nyстыx. Понятие $\alpha A(\alpha)$ логически универсально, если предикат $A(\alpha)$ логически истинен, а его логическая форма представляет собой общезначимую формулу (например, «человек, умеющий плавать или не умеющий плавать»). Понятие $\alpha A(\alpha)$ логически пусто, если предикат $A(\alpha)$ логически ложен, а его логическая форма представляет собой невыполнимую формулу (например, «человек, умеющий плавать и не умеющий плавать»). 
Также Е.К. Войшвилло было существенно уточнено основание деления понятий по структуре элементов их объема: дано различение понятий об отдельно взятых объектах (они имеют вид $\alpha A(\alpha))$ и понятий о кортежах объектов (имеющих вид $\left.\alpha_{1} \alpha_{2} \ldots \alpha_{n} A\left(\alpha_{1}, \alpha_{2}, \ldots, \alpha_{n}\right)\right)$. Среди понятий вида $\alpha A(\alpha)$ выделяются собирательные понятия: в них переменная $\alpha$ пробегает по множеству, элементами которого также являются множества.

По природе, онтологическому статусу обобщаемых объектов понятия принято делить на конкретные и абстрактные. Сам Войшвилло проводит данную дистинкцию лишь применительно к несобирательным понятиям вида $\alpha A(\alpha)$. Элементами объемов конкретных понятий являются индивиды (существа, вещества, государства, геометрические фигуры и т.п.), и на месте $\alpha$ в знаковом представлении таких понятий используются предметные (индивидные) переменные. Что же касается абстрактных понятий, то в них обобщаются не индивиды, а характеристики (свойства, отношения, предметно-функциональные зависимости), и потому в качестве $\alpha$ при их записи в языке используются предикаторные или предметно-функциональные переменные.

В принципе, различение конкретных и абстрактных понятий можно распространить и на понятия с иными структурами элементов объема, а именно на собирательные (понятия о множествах) и понятия о кортежах объектов. Так, в [8, с. 286] конкретными называются понятия, элементами объемов которых являются индивиды (например, «электропроводное вещество»), кортежи индивидов (например, «мужчины, имеющие общих родителей») или множества индивидов (например, «пучок параллельных прямых»). K абстрактным следует отнести понятия, элементами объемов которых являются отдельные характеристики (например, «способность вещества проводить электричество»), кортежи характеристик (напр., «противоположные человеческие качества») или множества характеристик (например, понятие фенотипа - «совокупность всех свойств строения и жизнедеятельности организма, обусловленных взаимодействием его генотипа с условиями среды»).

В заключение остановлюсь вкратце еще на двух важных результатах Е.К. Войшвилло, связанных с его учением о понятии. 
Первый заключается в трактовке научных аксиоматических теорий как понятий. Действительно, ряд теорий можно представить в виде конструкций вида $\left.\alpha_{1} \alpha_{2} \ldots \alpha_{n} A\left(\alpha_{1}, \alpha_{2}, \ldots, \alpha_{n}\right)\right)$, например, алгебраические системы (решетки, алгебры, поля, кольца и т.п.) часто определяются как «кортежи, состоящие из множества и заданных на нем свойств, отношений и операций, которые обладают некими характеристиками, фиксирующимися в аксиомах данной теории». Такой подход может дать новый, оригинальный взгляд на вопрос о познавательном статусе теорий, их структуре, а также на проблемы метатеоретических отношений между ними.

Суть второго результата состоит в применении современного варианта учения о понятии к центральной проблематике логики как науки, а именно к теории дедукции. Е.К. Войшвилло разработал специальный формализованный язык, использующий понятийные конструкции типа $\alpha A(\alpha)$ и построил в нем формальное исчисление [2]. Этот язык, по мнению Войшвилло, ближе к естественному, нежели язык стандартного исчисления предикатов. Действительно, категорические высказывания выражаются здесь, как и в естественном языке, без использования бинарных пропозициональных связок $(\supset$ и $\wedge$ ). Например, общеутвердительное высказывание «Всякий $S$ есть $P$ » записывается как $\forall x S(x) P(x S(x))$, а частноотрицательное высказывание «Некоторый $S$ не есть $P »$ как $\exists x S(x) \neg P(x S(x))$. Предложенное Е.К. Войшвилло исчисление требует дальнейшего исследования современными металогическими средствами.

\section{Литература}

[1] Бочаров В.А., Маркин В.И. Основы логики. М.: ИД «Форум»Инфра-М, 2011.

[2] Войшвилло E.К. Опыт построения исчисления предикатов, приближенного к естественному языку // Логическая структура научного знания. М.: Наука, 1965.

[3] Войшвилло E.K. Понятие. М.: Издательство Московского университета, 1967.

[4] Войшвилло E.K. Понятие (в формальной логике) // Философская энциклопедия. Т. 4. М.: Советская Энциклопедия, 1967. С. 316-318. 
[5] Войшвилло E.K. Понятие как форма мышления: логикогносеологический анализ. М.: Издательство Московского университета, 1989.

[6] Войшвилло E.K., Дегтярев М.Г. Логика как часть теории познания и научной методологии. Фундаментальный курс. Книга II. М.: Наука, 1994.

[7] Ивлев Ю.В. Логика. Учебник для студентов философских и юридических специальностей. М.: Проспект, 2012.

[8] Маркин В.И. Понятие // Новая философская энциклопедия. Том третий. М.: Мысль, 2010. С. 185-187

[9] Смирнова Е.Д. Логика и философия. М.: РОССПЭН, 1996.

\section{References (transliteration)}

[1] Bocharov V.A., Markin V.I. Osnovy logiki. M.: ID Forum - Infra-M, 2011.

[2] Vojshvillo E.K. Opyt postroenija ischislenija predikatov, priblizhennogo k estestvennomu jazyku // Logicheskaja struktura nauchnogo znanija. M.: Nauka, 1965.

[3] Vojshvillo E.K. Ponjatie. M.: Izdanie moskovskogo universiteta, 1967.

[4] Vojshvillo E.K. Ponjatie (v formal'noj logike) // Filosofskaja enciklopedija. Tom. 4. M.: Sovetskaja enciklopedija, 1967. S. 316-318.

[5] Vojshvillo E.K.Ponjatie kak forma myshlenija: logiko-gnoseologicheskij analiz. M.: Izdanie moskovskogo universiteta, 1989.

[6] Vojshvillo E.K., Degtjarev M.G. Logika kak chast' nauchnogo poznanija i nauchnoj metodologii. Fudamental'nyj kurs. Kniga II. M.: Nauka, 1994.

[7] Ivlev Ju. V. Logika. Uchebnik dlja studentov filosofskih i juridicheskih special'nostej. M.: Prospekt, 2012.

[8] Markin V.I. Ponjatie // Novaja filosofskaja enciklopedija. Tom tretij. M.: Mysl', 2010. S. 185-187

[9] Smirnova E.D. Logika i filosofija. M.: ROSSPEN, 1996. 